\title{
PREPARATION AND ANTIMICROBIAL STUDIES OF NOVEL FUSED HETEROCYCLIC COMPOUNDS
}

\author{
Rekha Khairwar $^{1}$, Diwa Mishra ${ }^{1}$ and Arun Singh ${ }^{2, 凶}$ \\ ${ }^{1}$ Department of Chemistry, Govt. Geetanjali Girls' PG College Bhopal, India \\ ${ }^{2}$ Department of Chemistry, Govt. Maharani Laxmibai Girls' PG College Bhopal, India \\ Corresponding Author: dr.arunsingh68@gmail.com
}

\begin{abstract}
The imidazole derivate say 2-(1-methyl-1H-benzo[d]imidazol-2-ylthio)acetohydrazide (1) was synthesized. Various Schiff bases (3a-e) of (1) were prepared by reacting with various benzaldehyde derivates (2a-e). All the 3a-e compounds reacted with Succinic Anhydride to afford 1-(2-(1-methyl-1H-benzo[d]imidazol-2-ylthio) acetamido)-5oxo-2-arylpyrrolidine-3-carboxylic acid derivatives (4a-e). These (4a-e) compounds reacted with o-phenylene diamine yields N-(3-(1H-benzo[d]imidazol-2-yl)-5-oxo-2-arylpyrrolidin-1-yl)-2-(1-methyl-1H-benzo[d]imidazol-2ylthio) acetamide (5a-e). The Compounds (5a-e) reacted with benzaldehyde affords 4-benzylidene derivates(6ae), they further reacted with hydrazine hydrate gives N-(4-(1H-benzo[d]imidazol-2-yl)-5-aryl-3-phenyl-4,5dihydropyrrolo[2,3-c]pyrazol-6(2H)-yl)-2-((1-methyl-1H-benzo[d]imidazol-2-yl)thio)acetamide (7a-e).All the synthesized compounds characterized spectroscopically and tested for antimicrobial activity.

Keywords: Benzimidazole, Pyrrolidine, Isoxazole, Characterization, Antibacterial activity, and Antifungal activities.
\end{abstract}

RASĀYAN J. Chem., Vol. 14, No.3, 2021

\section{INTRODUCTION}

Heterocyclic compounds exhibit pharmaceutical as well as biological activity. Among them, Benzimidazole derivatives have been found to possess various biological as well as pharmacological activities, such as anti-inflammatory, analgesics, antipyretic and antifungal, anticonvulsant, antitumor, antiviral and analgesic activities. ${ }^{1-7}$ Pyrazole display various activity like antibacterial, antifungal, antimicrobial, antidepressant, anti-tumor, anticonvulsant, anti-inflammatory and anti-amoebic activities. ${ }^{8-}$ 15

The present research paper deals with the synthesis of novel heterocyclic compounds, N- $(4-(1 \mathrm{H}-$ benzo[d]imidazol-2-yl)-5-aryl-3-phenyl-4,5-dihydropyrrolo[2,3-c]pyrazol-6(2H)-yl)-2-((1-methyl-1Hbenzo[d]imidazol-2-yl)thio)acetamide which contains benzimidazole and pyrazole. The synthetic route is as follows.

\section{EXPERIMENTAL}

\section{Materials}

All chemicals used were of laboratory grade. 2-(1-methyl-1H-benzo[d]imidazol-2-ylthio)acetohydrazide (1) prepared by reported research work. ${ }^{16}$

\section{Measurement}

Melting points were determined in open capillary tubes and were uncorrected. The IR spectra were recorded in $\mathrm{KBr}$ pellets on a Nicolet 400D spectrometer and ${ }^{1} \mathrm{H}$ NMR spectra were recorded in DMSO with TMS as internal standard on a Bruker spectrometer at $400 \mathrm{MHz}$.

Preparation of 1-(2-(1-methyl-1H-benzo[d]imidazol-2-ylthio) acetamido)-5-oxo-2-aryl pyrrolidine3-carboxylic acid derivatives (4a-e)

A stoichiometric amount of 2-(1-methyl-1H-benzo[d]imidazol-2-ylthio)acetohydrazide (1) and substituted benzaldehyde (2a-e) in absolute alcohol was heated on the steam bath for 3 hours. The solid separated was filtered, washed and then reacted with succinic anhydride in p-xylene was heated and on

Rasayan J. Chem., 14(3), 1947-1952(2021)

http://doi.org/10.31788/RJC.2021.1436365

This work is licensed under a CC BY 4.0 license. 
RASĀYAN J. Chem.

Vol. 14 | No. 3 |1947-1952| July - September | 2021

the steam bath for 7.5 hours. The resultant product was kept aside for 3 days. The obtained product was filtered and recrystallized by using rectified spirit and yielded 1-(2-(1-methyl-1H-benzo[d]imidazol-2ylthio) acetamido)-5-oxo-2-aryl pyrrolidine-3-carboxylic acid derivatives (4a-e). The yields, melting points and other characterization data of these compounds are given in Table -1 .

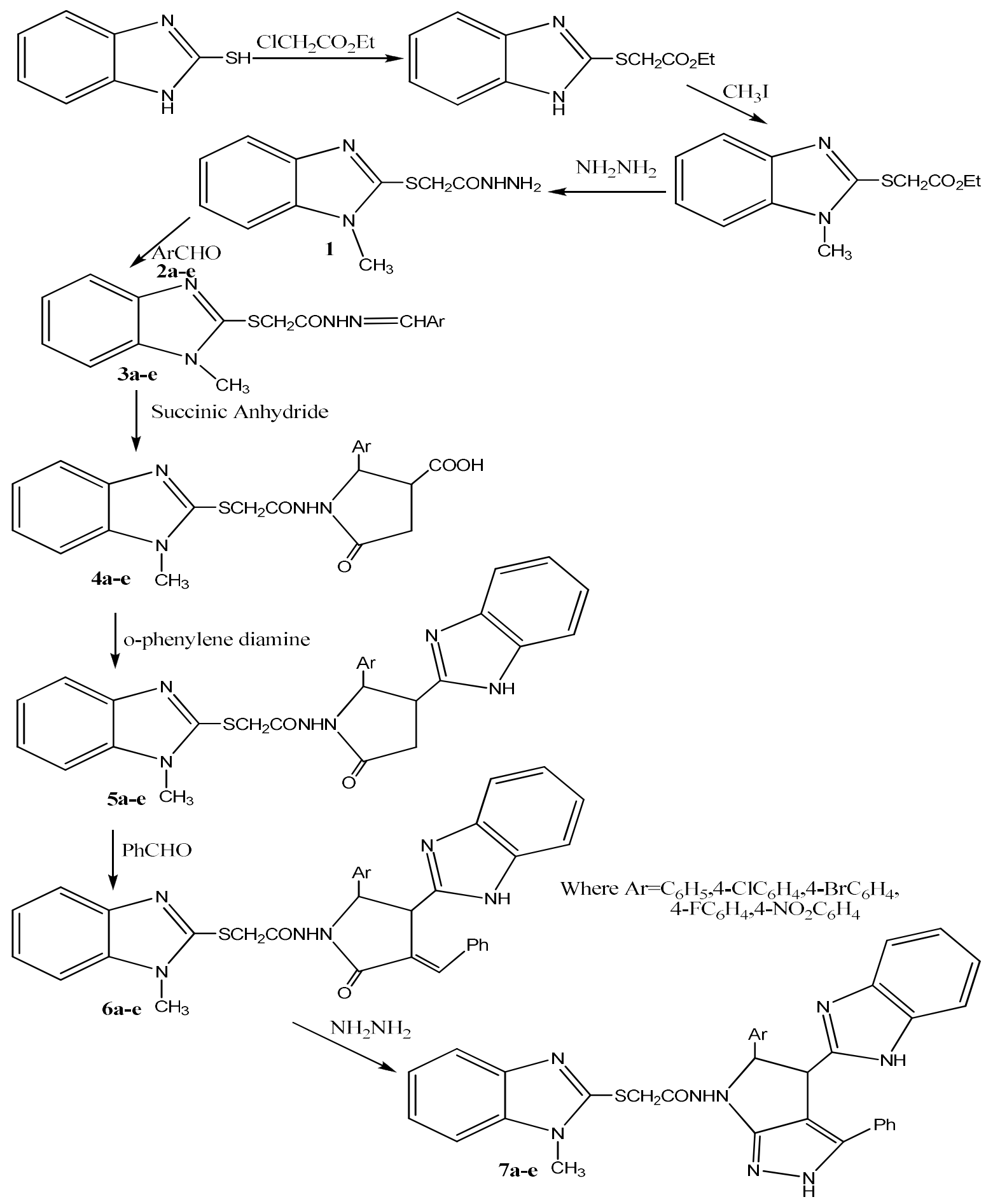

Scheme-1 
RASĀYAN J. Chem.

Vol. 14 | No. 3 |1947-1952| July - September | 2021

Table-1: Analytical Data and Elemental Analysis of Compounds (4a-e)

\begin{tabular}{|c|c|c|c|c|c|c|c|c|c|c|c|}
\hline \multirow{3}{*}{ Compd. } & \multirow{3}{*}{$\begin{array}{l}\text { Molecular formula } \\
\text { (Mol.wt.) }\end{array}$} & \multirow{3}{*}{$\begin{array}{l}\text { Yield } \\
\%\end{array}$} & \multirow{3}{*}{$\begin{array}{l}\text { M.P. }{ }^{*} \\
{ }^{0} \mathrm{C}\end{array}$} & \multicolumn{6}{|c|}{ Elemental Analysis } & \multirow{2}{*}{\multicolumn{2}{|c|}{$\% \mathrm{~S}$}} \\
\hline & & & & \multicolumn{2}{|c|}{$\% \mathrm{C}$} & \multicolumn{2}{|c|}{$\% \mathrm{H}$} & \multicolumn{2}{|c|}{$\% \mathrm{~N}$} & & \\
\hline & & & & Found & Calcd. & Found & Calcd. & Found & Calcd. & Found & Calcd. \\
\hline $4 a$ & $\begin{array}{c}\mathrm{C}_{21} \mathrm{H}_{20} \mathrm{~N}_{4} \mathrm{O}_{4} \mathrm{~S} \\
(424)\end{array}$ & 79 & 208-209 & 59.4 & 59.42 & 4.7 & 4.75 & 13.1 & 13.20 & 7.5 & 7.55 \\
\hline $4 b$ & $\begin{array}{c}\mathrm{C}_{21} \mathrm{H}_{19} \mathrm{~N}_{4} \mathrm{O}_{4} \mathrm{SCl} \\
(457.5)\end{array}$ & 74 & $203-204$ & 54.9 & 54.96 & 4.1 & 4.17 & 12.2 & 12.21 & 6.9 & 6.99 \\
\hline $4 \mathrm{c}$ & $\begin{array}{c}\mathrm{C}_{21} \mathrm{H}_{19} \mathrm{~N}_{4} \mathrm{O}_{4} \mathrm{SBr} \\
(502)\end{array}$ & 69 & $200-201$ & 50.0 & 50.11 & 3.7 & 3.80 & 11.1 & 11.13 & 6.3 & 6.37 \\
\hline $4 d$ & $\begin{array}{c}\mathrm{C}_{21} \mathrm{H}_{19} \mathrm{~N}_{4} \mathrm{O}_{4} \mathrm{SF} \\
(442)\end{array}$ & 72 & 196-198 & 56.9 & 57.00 & 4.3 & 4.33 & 12.6 & 12.66 & 7.2 & 7.25 \\
\hline $4 \mathrm{e}$ & $\begin{array}{c}\mathrm{C}_{21} \mathrm{H}_{19} \mathrm{~N}_{5} \mathrm{O}_{6} \mathrm{~S} \\
(469)\end{array}$ & 70 & $192-193$ & 53.7 & 53.73 & 4.0 & 4.08 & 14.9 & 14.92 & 6.8 & 6.83 \\
\hline
\end{tabular}

* Uncorrected LC-MS data of 4a-429,4c-508

Preparation of N-(3-(1H-benzo[d]imidazol-2-yl)-4-benzylidene-5-oxo-2-arylpyrrolidin-1-yl)-2-(1methyl-1H-benzo[d]imidazol-2-ylthio)acetamide (6a-e)

A mixture of o-Phenylene diamine and 1-(2-(1-methyl-1H-benzo[d]imidazol-2-ylthio) acetamido)-5-oxo2-aryl pyrrolidine-3-carboxylic acid derivatives (4a-e) was refluxed thermally. The reaction mixture was cooled and sodium hydroxide solution was added and then the crude product of (5a-e) was washed with ice-cold water and dissolved in boiling water for recrystallization, filtered and dried. The solution of (5ae) and benzaldehyde in dioxane $(50 \mathrm{~mL})$ in the presence of sodium ethoxide were refluxed for about 4-5 hrs. The resulting product was recrystallized from $\mathrm{R}$-sprite to yield compound $\mathrm{N}-(3-(1 \mathrm{H}-$ benzo[d]imidazol-2-yl)-4-benzylidene-5-oxo-2-arylpyrrolidin-1-yl)-2-(1-methyl-1H-benzo[d]imidazol-2ylthio)acetamide (6a-e). The yields, melting points and other characterization data of these compounds are given in Table -2 .

Table-2: Analytical Data and Elemental Analysis of Compounds (6a-e)

\begin{tabular}{|c|c|c|c|c|c|c|c|c|c|c|c|}
\hline \multirow{3}{*}{ Compd. } & \multirow{3}{*}{$\begin{array}{l}\text { Molecular Formula } \\
\text { (Mol.wt.) }\end{array}$} & \multirow{3}{*}{$\begin{array}{l}\text { Yield } \\
\%\end{array}$} & \multirow{3}{*}{${ }^{\mathrm{M} . \mathrm{P} .{ }^{*}}$} & \multicolumn{6}{|c|}{ Elemental Analysis } & \multirow{2}{*}{\multicolumn{2}{|c|}{$\% \mathrm{~S}$}} \\
\hline & & & & \multicolumn{2}{|c|}{$\% \mathrm{C}$} & \multicolumn{2}{|c|}{$\% \mathrm{H}$} & \multicolumn{2}{|c|}{$\% \mathrm{~N}$} & & \\
\hline & & & & Found & Calcd. & Found & Calcd. & Found & Calcd. & Found & Calcd. \\
\hline $6 a$ & $\begin{array}{c}\mathrm{C}_{34} \mathrm{H}_{28} \mathrm{~N}_{6} \mathrm{O}_{2} \mathrm{~S} \\
(584)\end{array}$ & 76 & $\begin{array}{l}231- \\
233\end{array}$ & 69.8 & 69.84 & 4.8 & 4.83 & 14.3 & 14.37 & 5.4 & 5.48 \\
\hline $6 \mathrm{~b}$ & $\begin{array}{c}\mathrm{C}_{34} \mathrm{H}_{27} \mathrm{~N}_{6} \mathrm{O}_{2} \mathrm{SCl} \\
(617.5)\end{array}$ & 72 & $\begin{array}{l}238- \\
239\end{array}$ & 65.9 & 65.96 & 4.3 & 4.40 & 13.5 & 13.57 & 5.1 & 5.18 \\
\hline $6 c$ & $\begin{array}{c}\mathrm{C}_{34} \mathrm{H}_{27} \mathrm{~N}_{6} \mathrm{O}_{2} \mathrm{SBr} \\
(662)\end{array}$ & 66 & $\begin{array}{l}243- \\
245\end{array}$ & 61.5 & 61.54 & 4.0 & 4.10 & 12.6 & 12.66 & 4.8 & 4.83 \\
\hline $6 \mathrm{~d}$ & $\begin{array}{c}\mathrm{C}_{34} \mathrm{H}_{27} \mathrm{~N}_{6} \mathrm{O}_{2} \mathrm{SF} \\
(602)\end{array}$ & 70 & $\begin{array}{l}237- \\
239\end{array}$ & 67.7 & 67.76 & 4.5 & 4.52 & 13.9 & 13.94 & 5.3 & 5.32 \\
\hline $6 e$ & $\begin{array}{c}\mathrm{C}_{34} \mathrm{H}_{27} \mathrm{~N}_{7} \mathrm{O}_{4} \mathrm{~S} \\
(629)\end{array}$ & 67 & $\begin{array}{l}235- \\
236\end{array}$ & 64.8 & 64.85 & 4.3 & 4.32 & 15.5 & 15.57 & 5.0 & 5.09 \\
\hline
\end{tabular}

Preparation of N-(4-(1H-benzo[d]imidazol-2-yl)-5-aryl-3-phenyl-4,5-dihydropyrrolo[2,3-c]pyrazol6(2H)-yl)-2-((1-methyl-1H-benzo[d]imidazol-2-yl)thio)acetamide (7a-e)

The reaction mixture of (6a-e) and hydrazine hydrate in glacialacetic acid was refluxed in a magnetic stirrer for 8-9 hrs. The completion of the reaction observed by TLC using ethyl acetate/hexane. The reaction mixture was cooled to room temperature and poured into ice-cold water, then neutralized by dilute $\mathrm{HCl}$. The obtained solid was filtered, washed with water, and recrystallized from ethanol. The yields, melting points and other characterization data of these compounds are given in Table- 3 .

\section{Antibacterial Activities}

The antibacterial activities of all the compounds were monitored against gram-positive and gram-negative bacteria (shown in Table-4). The agar cup plate method ${ }^{17-19}$ was adopted. The result was measured as 
RASĀYAN J. Chem.

Vol. 14 | No. 3 |1947-1952| July - September | 2021

inhibition zone in $\mathrm{mm}$. and presented in Table-4. Examination of the result reveals that compounds $4 \mathrm{~b}, 6 \mathrm{~b}$ and $7 \mathrm{~b}$ were more toxic for all bacteria while other compounds are toxic but less than that at $3 \mathrm{~b}, 6 \mathrm{~b}$, and $7 b$.

Table-3: Analytical Data and Elemental Analysis of Compounds (7a-e)

\begin{tabular}{|c|c|c|c|c|c|c|c|c|c|c|c|}
\hline \multirow{3}{*}{ Compd. } & \multirow{3}{*}{$\begin{array}{l}\text { Molecular } \\
\text { Formula } \\
\text { (Mol.wt.) }\end{array}$} & \multirow{3}{*}{$\begin{array}{l}\text { Yield } \\
\%\end{array}$} & \multirow{3}{*}{${ }^{\text {M.P. }}{ }^{*}{ }^{0} \mathrm{C}$} & \multicolumn{6}{|c|}{ Elemental Analysis } & \multirow{2}{*}{\multicolumn{2}{|c|}{$\% \mathrm{~S}$}} \\
\hline & & & & \multicolumn{2}{|c|}{$\% \mathrm{C}$} & \multicolumn{2}{|c|}{$\% \mathrm{H}$} & \multicolumn{2}{|c|}{$\% \mathrm{~N}$} & & \\
\hline & & & & Found & Calcd. & Found & Calcd. & Found & Calcd. & Found & Calcd. \\
\hline $7 a$ & $\begin{array}{c}\mathrm{C}_{34} \mathrm{H}_{28} \mathrm{~N}_{8} \mathrm{OS} \\
(597)\end{array}$ & 73 & $\begin{array}{l}268- \\
269\end{array}$ & 68.4 & 68.44 & 4.7 & 4.73 & 18.7 & 18.78 & 5.3 & 5.37 \\
\hline $7 b$ & $\begin{array}{c}\mathrm{C}_{34} \mathrm{H}_{27} \mathrm{~N}_{8} \mathrm{OSCl} \\
(631.5)\end{array}$ & 70 & $\begin{array}{l}272- \\
273\end{array}$ & 64.6 & 64.70 & 4.3 & 4.31 & 17.7 & 17.75 & 5.0 & 5.08 \\
\hline $7 \mathrm{c}$ & $\begin{array}{c}\mathrm{C}_{34} \mathrm{H}_{27} \mathrm{~N}_{8} \mathrm{OSBr} \\
(675)\end{array}$ & 65 & $\begin{array}{l}267- \\
268\end{array}$ & 60.4 & 60.44 & 4.0 & 4.03 & 16.5 & 16.59 & 4.7 & 4.75 \\
\hline $7 d$ & $\begin{array}{c}\mathrm{C}_{34} \mathrm{H}_{27} \mathrm{~N}_{8} \mathrm{OSF} \\
(615)\end{array}$ & 68 & $\begin{array}{l}271- \\
272 \\
\end{array}$ & 66.4 & 66.43 & 4.4 & 4.43 & 18.2 & 18.23 & 5.2 & 5.22 \\
\hline $7 e$ & $\begin{array}{c}\mathrm{C}_{34} \mathrm{H}_{27} \mathrm{~N}_{9} \mathrm{O}_{3} \mathrm{~S} \\
(642)\end{array}$ & 63 & $\begin{array}{l}264- \\
265\end{array}$ & 63.6 & 63.64 & 4.2 & 4.24 & 19.6 & 19.64 & 4.9 & 5.00 \\
\hline
\end{tabular}

Table-4: Antibacterial Properties of Compounds (4a-e),(6a-e) and (7a-e)

\begin{tabular}{c|c|c|c|c}
\hline \multirow{2}{*}{ Compounds } & \multicolumn{2}{|c|}{ Gram +Ve } & \multicolumn{2}{c}{ Gram -Ve } \\
\cline { 2 - 5 } & $\begin{array}{c}\text { Bacillus } \\
\text { subtilis }\end{array}$ & $\begin{array}{c}\text { Staphylococcus } \\
\text { Aureus }\end{array}$ & $\begin{array}{c}\text { Klebsiella } \\
\text { promioe }\end{array}$ & E.coli \\
\hline $4 \mathrm{a}$ & 09 & 11 & 10 & 09 \\
\hline $4 \mathrm{~b}$ & 14 & 15 & 13 & 15 \\
\hline $4 \mathrm{c}$ & 11 & 12 & 8 & 11 \\
\hline $4 \mathrm{~d}$ & 12 & 13 & 12 & 13 \\
\hline $4 \mathrm{e}$ & 11 & 11 & 9 & 12 \\
\hline $6 \mathrm{a}$ & 12 & 15 & 11 & 11 \\
\hline $6 \mathrm{~b}$ & 17 & 19 & 14 & 17 \\
\hline $6 \mathrm{c}$ & 14 & 16 & 9 & 13 \\
\hline $6 \mathrm{~d}$ & 15 & 17 & 13 & 15 \\
\hline $6 \mathrm{e}$ & 14 & 15 & 10 & 14 \\
\hline $7 \mathrm{a}$ & 14 & 17 & 15 & 14 \\
\hline $7 \mathrm{~b}$ & 19 & 21 & 18 & 20 \\
\hline $7 \mathrm{c}$ & 16 & 18 & 13 & 16 \\
\hline $7 \mathrm{~d}$ & 17 & 19 & 17 & 18 \\
\hline $7 \mathrm{e}$ & 16 & 17 & 14 & 17 \\
\hline
\end{tabular}

\section{Antifungal Activities}

The antifungal activity of all three series of compounds was monitored for pathogens shown in Table- 5 . The method ${ }^{17-19}$ was adopted by using Potato-Dextrose -Agar (PDA) medium. The percentage of inhibitory growth of the fungus was measured from the area of colony growth and inhibitory. The result is shown in Table- 4 . The result indicates that compare $4 \mathrm{~b}, 6 \mathrm{~b}$ and $7 \mathrm{~b}$ are more toxic due to the presence of chlorine atoms in the molecules. The fungicidal activity of all the compounds was studied at $1000 \mathrm{ppm}$ concentration in vitro.

\begin{tabular}{c|c|c|c|c}
\multicolumn{5}{c}{ Table-5: Percentage of Inhibition Growth of Fungi by Compounds (4a-e),(6a-e) and (7a-e) } \\
\hline Compounds & $\begin{array}{c}\text { Nigrospora } \\
\text { Sp. }\end{array}$ & $\begin{array}{c}\text { Aspergillus } \\
\text { Niger }\end{array}$ & BotrydepladiaThiobromine & $\begin{array}{c}\text { Rhizopus } \\
\text { Nigricum }\end{array}$ \\
\hline 4a & 54 & 53 & 50 & 55 \\
\hline $4 \mathrm{~b}$ & 61 & 66 & 69 & 65 \\
\hline $4 \mathrm{c}$ & 63 & 65 & 66 & 69 \\
\hline $4 \mathrm{~d}$ & 56 & 61 & 64 & 56 \\
\hline $4 \mathrm{e}$ & 58 & 55 & 53 & 57 \\
\hline $6 \mathrm{a}$ & 58 & 55 & 53 & 57 \\
\hline
\end{tabular}


RASĀYAN J. Chem.

Vol. 14 | No. 3 |1947-1952| July - September | 2021

\begin{tabular}{c|c|c|c|c}
\hline $6 \mathrm{~b}$ & 65 & 68 & 72 & 67 \\
\hline $6 \mathrm{c}$ & 67 & 67 & 69 & 71 \\
\hline $6 \mathrm{~d}$ & 60 & 63 & 67 & 58 \\
\hline $6 \mathrm{e}$ & 62 & 57 & 56 & 59 \\
\hline $7 \mathrm{a}$ & 60 & 56 & 54 & 59 \\
\hline $7 \mathrm{~b}$ & 66 & 68 & 74 & 68 \\
\hline $7 \mathrm{c}$ & 66 & 66 & 70 & 65 \\
\hline $7 \mathrm{~d}$ & 61 & 65 & 69 & 59 \\
\hline $7 \mathrm{e}$ & 63 & 58 & 57 & 60 \\
\hline
\end{tabular}

RESULTS AND DISCUSSION

It was found that 2-(1-methyl-1H-benzo[d]imidazol-2-ylthio)acetohydrazide (1) undergoes facile condensation with substituted benzaldehyde(2a-e) to afford the corresponding Schiff's bases(3a-e). The (3a-e) react with succinic anhydride to gave 1-(2-(1-methyl-1H-benzo[d]imidazol-2-yl thio) acetamido)5-oxo-2-aryl pyrrolidine-3-carboxylic acid derivatives (4a-e), the structure of (4a-e) were confirmed by elemental analysis and IR spectra showing an absorption band at $1735 \mathrm{~cm}^{-1}(\mathrm{C}=\mathrm{O}$ of pyrrolidine ring), $3040-3058 \mathrm{~cm}^{-1}$ (C-H, of Ar.), 1650-1670 $\mathrm{cm}^{-1}$ (-CO of $\left.-\mathrm{COOH},-\mathrm{CONH}\right), 1620-1640(\mathrm{C}=\mathrm{N}), 710-720(\mathrm{C}-$ $\mathrm{S}), \quad 1080(-\mathrm{Cl}), 1555, \quad 1375 \quad\left(-\mathrm{NO}_{2}\right), \quad 710(\mathrm{C}-\mathrm{Br}), \quad 1255(\mathrm{C}-\mathrm{F}) . \quad{ }^{1} \mathrm{H} \quad \mathrm{NMR}: \quad 7.28-7.66 \quad$ (9H,m,Ar$\mathrm{H}), 5.15\left(1 \mathrm{H}, \mathrm{d}, \mathrm{C}_{2} \mathrm{H}\right.$ of the ring $), 3.37\left(1 \mathrm{H}, \mathrm{q}, \mathrm{C}_{3} \mathrm{H}\right.$ of the ring $), 2.55,2.79\left(2 \mathrm{H}, \mathrm{d}, \mathrm{C}_{4} \mathrm{H}\right.$ of the ring $), 12.9(1 \mathrm{H}, \mathrm{s},-$ $\mathrm{COOH}), 11.80(1 \mathrm{H}, \mathrm{s},-\mathrm{CONH}), 4.02\left(2 \mathrm{H}, \mathrm{s},-\mathrm{CH}_{2}\right), 3.84\left(3 \mathrm{H}, \mathrm{s},-\mathrm{CH}_{3}\right)$. The $\mathrm{C}, \mathrm{H}, \mathrm{N}$ analysis data of all compounds are presented in Table-1.

The structures assigned to N-(3-(1H-benzo[d]imidazol-2-yl)-4-benzylidene-5-oxo-2-arylpyrrolidin-1-yl)2-(1-methyl-1H-benzo[d]imidazol-2-ylthio)acetamide (6a-e) were supported by the elemental analysis and IR spectra showing an absorption bands at $1735 \mathrm{~cm}^{-1}(\mathrm{C}=\mathrm{O}$ of pyrrolidine ring $), 3040-3058 \mathrm{~cm}^{-1}(\mathrm{C}-\mathrm{H}$, of Ar.),3200-3220 cm $\mathrm{cm}^{-1}(\mathrm{~N}-\mathrm{H}), 1625 \mathrm{~cm}^{-1}(-\mathrm{C}=\mathrm{CH}-\mathrm{Ar}), 1650-1670 \mathrm{~cm}^{-1}$ (-CO of $\left.-\mathrm{CONH}\right), 1620-1640$ $(\mathrm{C}=\mathrm{N}), 710-720(\mathrm{C}-\mathrm{S}), \quad 1080(-\mathrm{Cl}), 1555,1375 \quad\left(-\mathrm{NO}_{2}\right), \quad 710(\mathrm{C}-\mathrm{Br}), \quad 1255(\mathrm{C}-\mathrm{F}) . \quad{ }^{1} \mathrm{H} \quad \mathrm{NMR}: \quad 7.28-$ $7.68(14 \mathrm{H}, \mathrm{m}, \mathrm{Ar}-\mathrm{H}), 5.15\left(1 \mathrm{H}, \mathrm{d}, \mathrm{C}_{2} \mathrm{H}\right.$ of the ring $), 4.80\left(1 \mathrm{H}, \mathrm{q}, \mathrm{C}_{3} \mathrm{H}\right.$ of the ring $), 11.80(1 \mathrm{H}, \mathrm{s},-\mathrm{CONH})$, $4.02\left(2 \mathrm{H}, \mathrm{s},-\mathrm{CH}_{2}\right), 3.84\left(3 \mathrm{H}, \mathrm{s},-\mathrm{CH}_{3}\right), 7.30,7.52(2 \mathrm{H}, \mathrm{s},=\mathrm{CH}), 5.48(1 \mathrm{H}, \mathrm{s},-\mathrm{NH})$. The $\mathrm{C}, \mathrm{H}, \mathrm{N}, \mathrm{S}$ analysis data of all compounds are presented in Table-2. The structures assigned to N-(4-(1H-benzo[d]imidazol-2-yl)-5aryl-3-phenyl-4,5-dihydro pyrrole [2,3-c]pyrazol-6(2H)-yl)-2-((1-methyl-1H-benzo[d]imidazol-2yl)thio)acetamide (7a-e) were supported by the elemental analysis and IR spectra showing an absorption bands at $1735 \mathrm{~cm}^{-1}\left(\mathrm{C}=\mathrm{O}\right.$ of pyrrolidine ring), 3040-3058 $\mathrm{cm}^{-1}(\mathrm{C}-\mathrm{H}$, of Ar. $), 3200-3220 \mathrm{~cm}^{-1}(\mathrm{~N}-\mathrm{H}), 1625$ $\mathrm{cm}^{-1}(-\mathrm{C}=\mathrm{CH}), 1650-1670 \mathrm{~cm}^{-1}$ (-CO of $\left.-\mathrm{CONH}\right), 1620-1640(\mathrm{C}=\mathrm{N}), 710-720(\mathrm{C}-\mathrm{S}), 1080(-\mathrm{Cl}), 1555,1375$ $\left(-\mathrm{NO}_{2}\right), 710(\mathrm{C}-\mathrm{Br}), 1255(\mathrm{C}-\mathrm{F}) .{ }^{1} \mathrm{H}$ NMR: 7.28-8.16 $(18 \mathrm{H}, \mathrm{m}, \mathrm{Ar}-\mathrm{H}), 4.75-4.80(2 \mathrm{H}, \mathrm{d}, \mathrm{CH}$ of the ring), $11.80(1 \mathrm{H}, \mathrm{s},-\mathrm{CONH}), 7.90,5.48(2 \mathrm{H}, \mathrm{s},-\mathrm{NH}) .4 .02\left(2 \mathrm{H}, \mathrm{s},-\mathrm{CH}_{2}\right), 3.84\left(3 \mathrm{H}, \mathrm{s},-\mathrm{CH}_{3}\right)$. The $\mathrm{C}, \mathrm{H}, \mathrm{N}$ analysis data of all compounds are presented in Table-3.

The examination of elemental analytical data reveals that the elemental contents are consistent with the predicted structure shown in Scheme-1. The IR data are also directing for the assignment of the predicted structure.

\section{CONCLUSION}

In present research work, we synthesis novel fused heterocyclic compound say, N- $(4-(1 \mathrm{H}-$ benzo[d]imidazol-2-yl)-5-aryl-3-phenyl-4,5-dihydropyrrolo[2,3-c]pyrazol-6(2H)-yl)-2-((1-methyl-1H-

benzo[d]imidazol-2-yl)thio)acetamide (7a-e) from 4-benzylidene derivates of $\mathrm{N}-(3-(1 \mathrm{H}-$ benzo[d]imidazol-2-yl)-5-oxo-2-arylpyrrolidin-1-yl)-2-(1-methyl-1H-benzo[d]imidazol-2-ylthio)

acetamide (5a-e) and hydrazine hydrate. All the novel synthesized compounds characterized spectroscopically, which assign the structure of synthesized compounds and tested for antimicrobial activity shows moderate to good toxicity.

\section{REFERENCES}

1. J. R. Sharma, Heterocyclic Letters,7(2), 405(2017), https://doi.org/10.7897/2230-8407.087112.

2. H.J.Al-Karagully and A.A.RazakMahamood,Journal Pharmacy Science \& Research, 9(2), 260(2017), https://doi.org/10.214/0929867325621.80228164656 
RASĀYAN J. Chem.

Vol. 14 | No. 3 |1947-1952| July - September | 2021

3. P. J. Sharma, Organic Chemistry-An Indian Journal, 11(2), 77(2015), https://doi.org/10.3762/Ocaij.2.15.

4. R. V. Heralagi, K. N. Jayavera and B. Shivkumar, Research Journal of Pharmaceutical, Biological and Chemical Sciences, 3(2), 407 (2015), https://doi.org/10.1080/14756366.2017.1417276

5. S. Giri and K. M.Basavaraja, Journal of Chemistry and Pharmacy Research , 4(5), 2643(2012), https://doi.org/10.11648/Ajhc.20190502.12.20.

6. B. Sarkar, R. Shukla, S. Shukla and S. Kumar, Asian Journal Pharmacy Research, 4(3),118 (2014), https://doi.org/10.30750/ajpr.3.3.10

7. K. A. Al-Rashood and H. A. Abdel-Aziz, Molecules, 15, 3775(2010), https://doi.org/10.3390/molecules20058800

8. P. Y. Rajendra, R. A. Lakshmana, L. Prasoona, K. Murali, K. P. Ravi, Bioorganic Medicinal Chemistry Letter, 15, 5030(2005), https://doi.org/10.1016/j.bmcl.2005.08.040

9. Z. Ozdemir, H. B. Kandilici, B. Gumusel, U. Calis, A. A. Bilgin, European Journal Medicinal Chemistry, 42, 373(2007), https://doi.org/10.1016/j.ejmech.2006.09.006

10. G. T. Zitouni, A. Ozdemir, K. Guven, Archives of Pharmaceutical (Weinheim).,338, 96 (2005), https://doi.org/10.1002/ardp.200400935

11. A. Budakoti, M. Abid and A. Azam, European Journal of Medicinal Chemistry .,41,63(2006), https://doi.org/10.1016/j.ejmech.2005.06.013

12. E. C. Taylor and H. H. Patel, Tetrahedron, 48,8089(1992), https://doi.org/10.1016/S00404020(01)80479-8

13. O. A. Fathalla, M. E. Zaki, S. A. Swelam, S. M. Nofal, W. I. El-Eraky, Acta Polonie Pharmacy, 60,51(2003), https://doi.org/10.32383/appdr/130067

14. S. S. Korgaokar, P. H. Patil, M. J. Shah, H.H. Parekh, Indian Journal of Pharmacy Science, 58,222(1996), https://doi.org/10.1007/ Ijph111640131367.96.

15. J. G. Lombardino and I. G. Otterness, Journal of Medicinal Chemistry,24,830(2001), https://doi.org/10.1021/jm00139a012

16. S. A. Nevade, S. G. Lokapure and N. V. Kalyane, Journal of Korean Chemical Science ,57(6),755(2013), http://dx.doi.org/10.5012/jkcs.2013.57.6.755

17. C. H. Collins, Microbiological Methods, London, 364 (1967).

18. P. J. Shah, B. P. Patel and H. S. Patel, Journal of Saudai Chemical Society ,17(3),307(2013), https://doi.org/10.1016/j.jscs.2011.04.016

19. P. B. Godkar, Textbook of Medicinal Tech., Bhalani Publication House, Mumbai, pp. 326, 332,382(1996).

[RJC-6365/2021] 